\title{
Numerical analysis of selectively coated MMI structures for the application in optical sensor technology
}

\author{
Artur Szewczuk, Marek Błahut \\ Department of Optoelectronics, Silesian University of Technology, Akademicka 2A, 44-100 Gliwice
}

Received May 18, 2015; accepted June 26, 2015; published June 30, 2015

\begin{abstract}
In this work the numerical analysis is presented of an optical sensor based on multimode interference structures with a sensing layer which covers selectively a multimode waveguide. The analyzed gradient index optical waveguides are fabricated in a sodium-potassium ion exchange process. The effects of the sensing layer of different shape and excitation conditions are demonstrated.
\end{abstract}

Applications of multimode interference structures in waveguide sensor technology have been the subject of research studies in recent years. The principle of operation of each of these devices described in many scientific publications $[1,2]$ is similar. In most cases, the multimode sections are covered by layers whose optical parameters change when they are exposed to a measured medium. The modification of external physical parameters leads to changes in propagation conditions of light, and this effect is easily observable in the output signal. There is a wide range of cover material to choose from. It can be a simple dielectric medium [2] when the operation characteristic of the proposed sensor depends on the geometry and size of MMI structures. In a gradient index waveguide made by potassium-sodium ion exchange the effect strongly depends on the polarization of light [3]. When choosing thin metal oxides layers as the cover material [4] one can observe output signals dependence on both the refractive index and extinction coefficient changes. It is also possible to improve the characteristics of the sensor by the use of a very thin buffer layer tens of nanometers thick [5].

In this work a new configuration of MMI optical sensor with a sensing layer covering selectively a multimode waveguide is presented. It will be shown that the geometry of the sensing layer significantly affects the field distributions in the MMI section and therefore strongly influences the operation characteristics of the sensor.

The configuration of the interference sensor based on the selectively coated MMI structures is shown in Fig. 1. It includes an optical system composed of a single-mode waveguide, an MMI section which length is related to the distance of a single image formation, and a single-mode output waveguide. The MMI section is covered selectively by a sensing layer. The evanescent field of the modes propagating in a multimode waveguide penetrates the cladding. Changes of its refractive index have an impact on the mode properties of the multimode waveguide, as a result affecting the location of the input field image. The changes of image location are registered by a single-mode output waveguide. Two cases of input and output waveguide positions are considered symmetrical excitation (the case of symmetric interference) and excitation at one third of the MMI section width corresponding to paired interference.

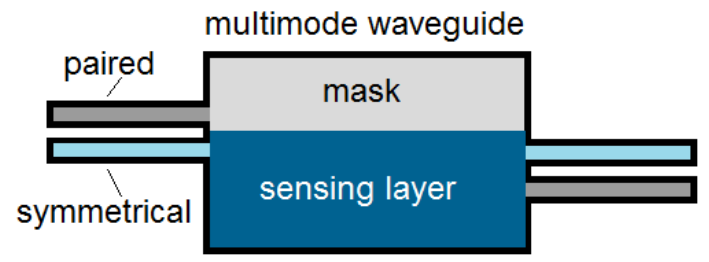

Fig. 1. Configuration of the sensor structure in the case of symmetrical and paired interference. Input single-mode waveguide for paired interference is situated at $1 / 3$ of the MMI section width and for symmetric interference - centrally.

The investigated MMI sections are produced by the sodium-potassium ion exchange process in a borosilicate glass $(n=1.5137)$ through the window of the $4 \mu \mathrm{m}$ width for a single mode waveguide and $45 \mu \mathrm{m}$ for an MMI section. The length of the multimode waveguide, chosen to obtain a single image at the output, is equal to $5150 \mu \mathrm{m}$ for symmetrical interference and $6900 \mu \mathrm{m}$ for paired. The distribution profile of the refractive index of the MMI section obtained in a diffusion process is calculated numerically from the nonlinear diffusion equation [3].

$$
\frac{\partial C_{K}}{\partial t}=\nabla\left[\frac{D_{K}}{1-(1-m) \cdot C_{K}} \cdot \nabla C_{K}\right]
$$

where $C_{K}$ is the dopant $\mathrm{K}^{+}$ions concentration, proportional to the refractive index change. The material parameters of the technological process - self-diffusion coefficient of $\mathrm{K}^{+}$ions $D_{K}$, the mobility ratio $m$ of the ions $\mathrm{K}^{+}$and $\mathrm{Na}^{+}$, and the maximum of the refractive index change were determined by measuring respective planar index profiles using the IWKB method. The determined self-diffusion coefficient is equal to $2.18 \mu \mathrm{m}^{2} / \mathrm{h}$, whereas the refractive index change distribution maximum at the surface is equal to 0.0095 . 
A gradient index MMI structure configuration with a non-uniform cladding is shown in Fig. 2. The MMI section is covered by a mask which selectively restricts the dimensions of the sensing layer. The mask partially covers the gradient index core of a multimode waveguide distributed in the glass substrate. It is assumed that the mask is made from a PMMA layer with the refractive index equal to 1.489 . It is also assumed in our calculations that the refractive index of the sensing layer varies from 1.33 to 1.49 .

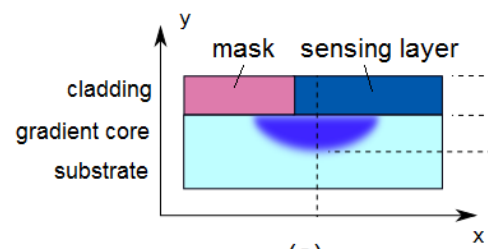

(a)

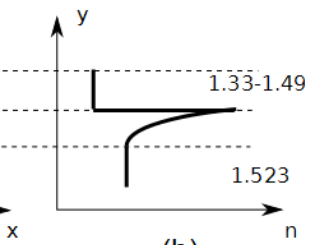

(b)
Fig. 2. Gradient index MMI structure configuration with a selective cladding (a) cross section of the MMI structure, (b) refractive index distribution for the vertical cutting marked in the figure by the dotted line. The refractive index of the sensing layer varies from 1.33 to 1.49.

Different configurations of considered masks are shown in Fig.3a, b. Also marked are the input waveguides positions at the beginning of the mask in the case of paired interference. The open parts for a rectangular mask are equal to $0.1,0.5$ and 0.9 of the waveguide width respectively. The shape of a triangular mask and its open parts are specified in Fig. 3b.

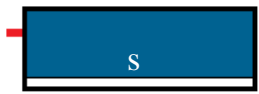

$0.1 \mathrm{~d}$

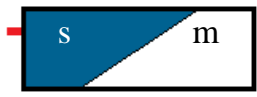

e

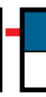

$0.5 \mathrm{~d}$

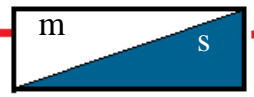

$f$

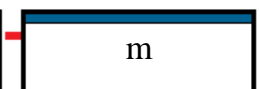

$0.9 \mathrm{~d}$
Fig. 3. Analyzed geometry of a cladding a) rectangular b) triangular. Symbol $\mathbf{s}$ denotes the sensing layer restricted by the mask and $\mathbf{m}$ denotes the mask shape.

Depending on the method of excitation, different interactions between the cladding and signal can be obtained.

The analyzed sensor measures the differences of the refractive index between the mask material and the sensing layer. The geometry of the sensing layer significantly affects modal field distributions in the MMI section and therefore strongly influences the operation characteristics of the modeled sensor. The difference of the refractive index between both parts of the cover results in a higher concentration of light energy in the area of a greater refractive index value. Field distribution changes are registered by a single-mode output waveguide.

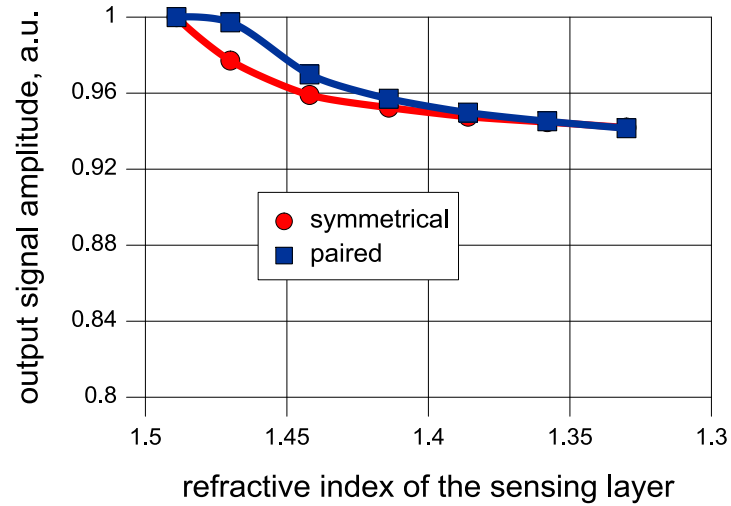

Fig. 4. Dependence of output signal amplitude on the refractive index of a sensing layer for symmetric and paired interference for an unmasked MMI structure.

The way of multimode waveguide excitation and the type of interference should be also significant for the working characteristics of designed structures.

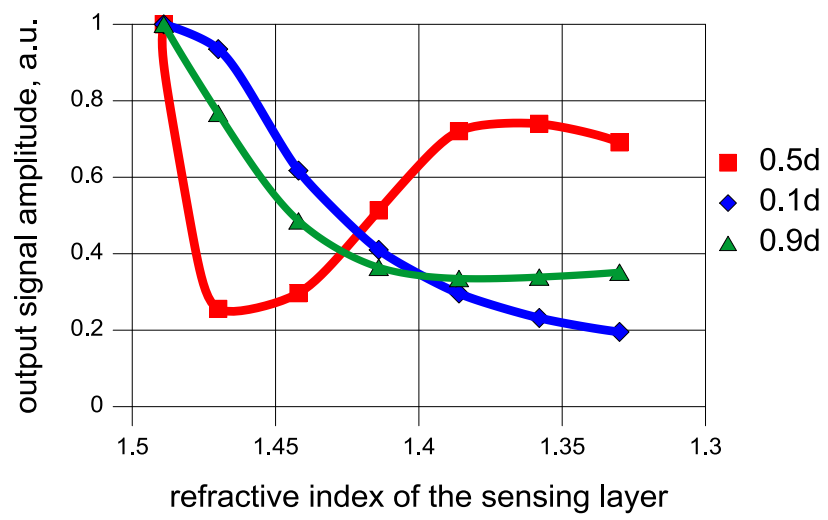

Fig. 5. Dependence of output signal amplitude on the refractive index of a rectangular sensing layer for symmetric interference. Different sizes of rectangular sensing layers are shown in Fig. 3a.

The calculations are performed by the 3D BPM method [4] for the wavelength $\lambda=0.635 \mu \mathrm{m}$ and gradient index profiles calculated numerically from Eq. (1). Signal amplitudes at the output for each configuration are determined as a function of the refractive index of the sensing layer. For comparison purposes the operation characteristics of an MMI sensor with a uniform sensing layer are calculated. Figure 4 presents output signal dependence on the refractive index of a uniform sensing layer for symmetric and paired interference. The characteristics change very slowly. The rate of changes is faster only for the refractive index of the cover near the maximum value of gradient distribution [4].

To improve operating characteristics, the selective sensing layers should be applied. In Figures 5-6 are shown output signal amplitudes in arbitrary units determined for different geometry of rectangular sensing layers defined in Fig. 3a, for symmetric interference and paired interference respectively. 


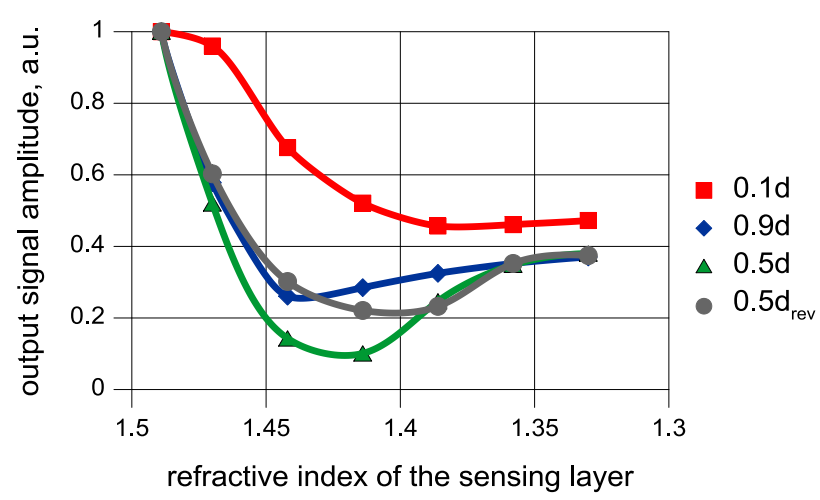

Fig. 6. Dependence of output signal amplitude on the refractive index of rectangular sensing layers for paired interference. Different sizes of rectangular sensing layers are shown in Fig. 3a.

In the case of symmetric interference for the mask opening of $0.5 \mathrm{~d}$ the structure is particularly sensitive to refractive index changes of the sensing layer close to the refractive index of the mask. On the other hand, the use of the mask openings equal to $0.1 \mathrm{~d}$ allows for the sensor operation in a wide range of refractive index changes.

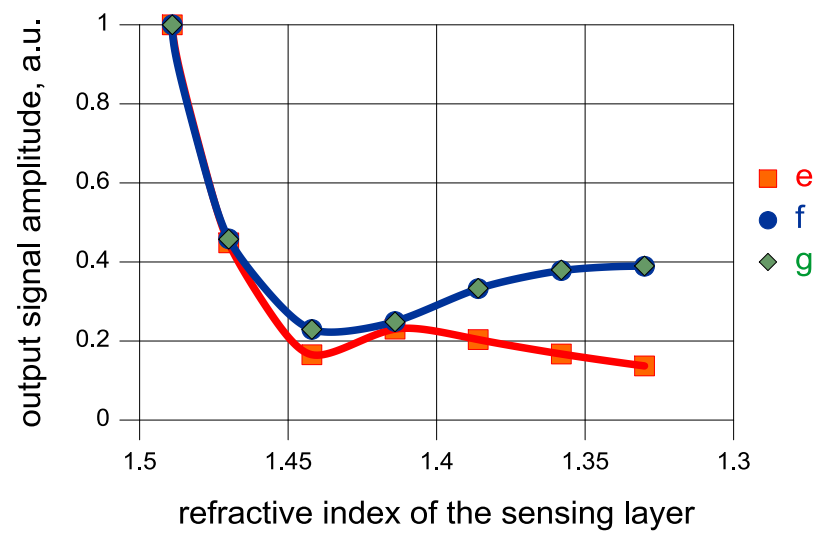

Fig. 7. Dependence of output signal amplitude on the refractive index of triangular sensing layers for symmetric interference. Different shapes of triangular sensing layers are shown in Fig.3b.

For paired interference, the sensing layer position should be determined in relation to the input single mode waveguide. The characteristic in Fig. 5 for $0.5 \mathrm{~d}$ describes the configuration when the sensing layer is situated in front of the input waveguide (Fig. 3a) and for $0.5 \mathrm{~d}_{\text {rev }}$ the input waveguide is in front of the mask. As before, the mask $0.5 \mathrm{~d}$ shows the biggest sensitivity to changes of the refractive index close to the refractive index of the mask but its operation range is wider.

The characteristics presented in Figs. 7-8 describe the influence of a triangular cladding for symmetric and paired interference. As can be seen from Fig. 7, the symmetric MMI structure does not distinguish $\mathrm{f}$ and $\mathrm{g}$ configurations and the sensitivity of the sensor is comparable with the previous case.

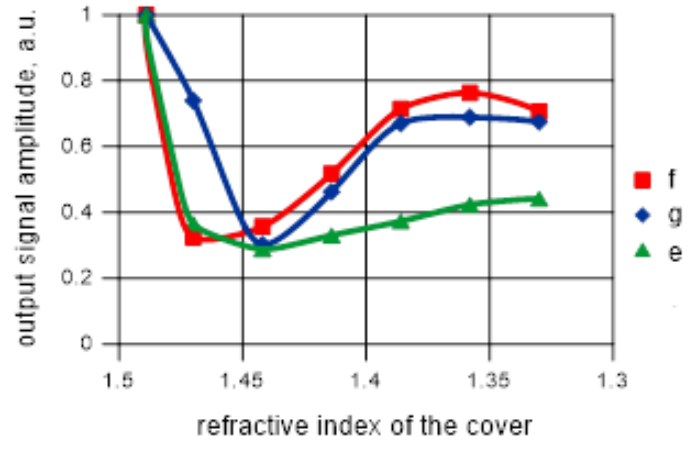

Fig. 8. Dependence of output signal amplitude on the refractive index of triangular sensing layers for paired interference. Different shapes of triangular sensing layers are shown in Fig. $3 b$.

It can be also noticed from Fig. 8 that MMI structures for paired interference are more sensitive to the changes of the triangular shape of the mask.

In summary, the geometry of the sensing layer significantly affects the field distributions in the MMI section and therefore strongly influences the operation characteristics of the modeled sensor. Numerical simulations of MMI sensors with a selective cover show that it is possible to change the operation characteristics of the sensor and adapt them to a specified measurement range. For selected geometry of the sensing layer we can measure, with a high sensitivity, the refractive index changes close to the refractive index of the mask and for another geometry of the sensing layer it is possible to specify its parameters in the whole analyzed range of the refractive index.

The work was partially sponsored by the Polish National Science Centre (NCN) within the grant 2012/07/B/ST7/01 471.

\section{References}

[1] K.R. Kribich et al., Sens. Actuat. B-Chem 107, 188 (2005)

[2] T. Mazingue et al., Opt. Comm., 278(2), 312 (2007).

[3] A. Szewczuk, M. Blahut, Optica Applicata, 43, 441 (2013).

[4] A. Szewczuk, M. Blahut, Acta. Phys. Pol. A 124, 570 (2013).

[5] A. Szewczuk, M. Blahut, Photonics Lett. Poland 6(4), 127 (2014). 